\title{
Calcular la fiabilidad de un cuestionario o escala mediante el SPSS: el coeficiente alfa de Cronbach
}

\author{
Julio Rodríguez-Rodríguez ${ }^{1}$ (D), Mercedes Reguant-Álvarez ${ }^{2}$
}

Artículo metodológico. Recibido: 31/10/2019. Aceptado:14/02/2020. Publicación avanzada 01/06/2020. Publicado: 01/07/2020

\section{Resumen}

La elaboración y aplicación de instrumentos de medida es una tarea habitual en la investigación socioeducativa. La utilización de cuestionarios, escalas y tests requiere que éstos sean instrumentos válidos y fiables. La fiabilidad, entendida como la precisión en la medida de una característica o un atributo, se puede calcular de diferentes maneras. El coeficiente alfa de Cronbach es uno de los habitualmente referenciados, y se puede utilizar en variables de escala. La finalidad de este artículo es proporcionar una información general que permita la comprensión de este índice y el procedimiento operativo para obtenerlo. El programa estadístico SPSS permite calcular este valor de manera relativamente fácil y ágil, contribuyendo así a la toma de decisiones relativas a la utilización del cuestionario o test, dado que la significación del coeficiente ha de tener en cuenta el contexto en el que se aplica. De esta manera, se logran los objetivos: identificar los diferentes métodos para calcular la fiabilidad de un instrumento en el ámbito socioeducativo; exponer los pasos para calcular el estadístico alfa de Cronbach mediante el paquete estadístico SPSS, y ejemplificar el cálculo de la fiabilidad de una escala de actitudes tipo Likert.

\section{Palabras clave}

Cuestionario; Fiabilidad; Medición; Investigación.

\section{Datos de los autores}

${ }^{1}$ Universitat de Barcelona, España. Profesor asociado de la Facultad de Educación (UB) y colaborador de la Universitat Oberta de Catalunya. También trabaja como educador social en el sistema de protección a la infancia. Es miembro del Grup de Recerca en Intervencions Socioeducatives en la Infància y la Joventut (GRISIJ). Contacto para la correspondencia: julio.rodriguez.ro@ub.edu

${ }^{2}$ Universitat de Barcelona, España. Profesora lectora del departamento de Métodos de Investigación y Diagnóstico en Educación, Facultad de Educación.

\section{Referencia recomendada}

Rodríguez-Rodríguez, J., y Reguant-Álvarez, M. (2020). Calcular la fiabilidad de un cuestionario o escala mediante el SPSS: el coeficiente alfa de Cronbach. REIRE Revista d'Innovació i Recerca en Educació, 13(2), 1-13. https://doi.org/10.1344/reire2020.13.230048

(C) 2020 Júlio Rodríguez-Rodríguez y Mercedes Reguant-Álvarez. Este artículo es de acceso abierto sujeto a la licencia Reconocimiento 4.0 Internacional de Creative Commons, la cual permite utilizar, distribuir y reproducir por cualquier medio sin restricciones siempre que se cite adecuadamente la obra original. Para ver una copia de esta licencia, visite http://creativecommons.org/licenses/by/4.0/ 


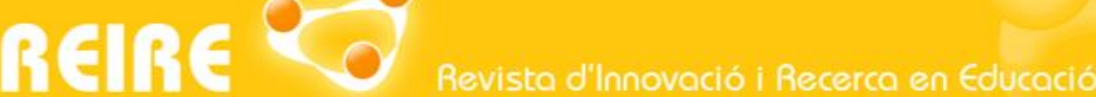

J. Rodríguez-Rodríguez y M. Reguant-Álvarez. Calcular la fiabilidad de un cuestionario o escala mediante el SPSS: el coeficiente alfa de Cronbach

Títol

Calcular la fiabilitat d'un qüestionari o escala mitjançant el SPSS: el coeficient alfa de Cronbach

Resum

L'elaboració i aplicació d'instruments de mesura és un treball habitual en la recerca socioeducativa. La utilització de qüestionaris, escales i tests demana que aquests instruments siguin instruments vàlids i fiables. La fiabilitat, entesa com la precisió en la mesura d'una característica o un atribut, es pot calcular a través de procediments diversos. El coeficient alfa de Cronbach és un mètode referenciat habitualment, i es pot utilitzar en opcions amb variables en escala. Així doncs, la finalitat de l'article és proporcionar una informació general que permeti la comprensió general d'aquest índex i el procediment operatiu per obtenir-lo. El programari estadístic SPSS permet calcular aquest valor de manera relativament fàcil i àgil, contribuint a la presa de decisions relatives a la utilització del qüestionari o test, ja que la significació del coeficient ha contemplar el context on s'aplica. D'aquesta manera, s'aconsegueixen els objectius proposats: identificar els diferents mètodes per calcular la fiabilitat d'un instrument en el context socioeducatiu; exposar els passos per calcular l'alfa de Cronbach mitjançant el programa estadístic SPSS, i exemplificar el càlcul de la fiabilitat d'una escala d'actituds tipus Likert.

\section{Paraules clau}

Qüestionari; Fiabilitat; Mesura; Investigació.

\section{Title}

Calculate the reliability of a questionnaire or scale using SPSS: Cronbach's alpha coefficient

\section{Abstract}

The development and application of measuring instruments is a common task in socio-educational research. The use of questionnaires, scales and tests requires that these be valid and reliable instruments. Reliability, understood as the accuracy in the measurement of a characteristic or an attribute, can be calculated in different ways. Cronbach's alpha coefficient is one of those usually referenced and can be used in scales variables. The purpose of this article is to provide general information that allows the understanding of this index and the operating procedure to obtain it. The statistical program SPSS allows to calculate this value in a relatively easy way, thus contributing to the decision making regarding the use of the questionnaire or test, since the significance of the coefficient has to take into account the context in which it is applied. In this way, the objectives are achieved: identify the different methods to calculate the reliability of an instrument in the socio-educational field; set out the steps to calculate Cronbach's alpha statistic using the SPSS statistical package, and exemplify the calculation of the reliability of a Likert-type attitude scale.

Keywords

Scale; Reliability; Measurement; Research 


\section{Introducción}

En la vida cotidiana, la medición está presente y resulta de gran utilidad. El consumo de agua de una familia, la temperatura corporal, o la velocidad de transmisión de datos a través de Internet son ejemplos diarios en los que tener una medición es una tarea útil y necesaria.

Sin embargo, en el ámbito de las Ciencias Humanas, la medición tiene unas características diferenciales en cuanto a otras disciplinas, ya que no se dispone de instrumentos con las características que posee un termómetro, un velocímetro o un barómetro, por poner algunos ejemplos. Así pues, el diseño y la construcción de instrumentos de medida es una tarea que no está exenta de complejidad. Como señalan López-Roldán y Fachelli $(2015$, p. 6):

La medición es pasar de los conceptos teóricos a los indicadores empíricos, por lo que se debe dar una correspondencia entre los conceptos que aluden a una realidad y la medición en una realidad, entre el lenguaje de los conceptos y el lenguaje de los números.

De esta manera, la medición tiene que ver con asignar valores alfanuméricos a una característica o propiedad observable en un fenómeno determinado a través de unos indicadores (Reguant y MartínezOlmo, 2014).

A la hora de asignar un carácter numérico o un símbolo a esa característica que se quiere medir, se suele recurrir a las escalas de medida. La clasificación habitual de las escalas de medida distingue cuatro tipos, según sirvan para realizar mediciones cualitativas o categóricas, o bien para realizar mediciones cuantitativas o continuas. Estos tipos, en orden ascendente de precisión, son los que se muestran en la Tabla 1.

Tabla 1

Escalas de medida

\begin{tabular}{|c|c|c|c|}
\hline Escala & Definición & Ejemplo & Estadísticos \\
\hline $\begin{array}{l}\text { Nominal } \\
\text { (cualitativa) }\end{array}$ & $\begin{array}{l}\text { Consiste en asignar dos o más números o } \\
\text { símbolos a los distintos valores de ese } \\
\text { atributo, con la finalidad de diferenciarlos } \\
\text { entre sí. La asignación de estos caracteres } \\
\text { es exhaustiva y mutuamente excluyente. } \\
\text { No lleva asociada ninguna idea de cantidad, } \\
\text { ni orden o jerarquía }\end{array}$ & $\begin{array}{l}\text { El género de una persona, } \\
\text { que podría escalarse como: } \\
1=\text { masculino } \\
2 \text { = femenino } \\
3=\text { no binario }\end{array}$ & $\begin{array}{l}\text { Frecuencia } \\
\text { Moda } \\
\text { Porcentajes }\end{array}$ \\
\hline $\begin{array}{l}\text { Ordinal } \\
\text { (cualitativa) }\end{array}$ & $\begin{array}{l}\text { La asignación de números lleva implícita } \\
\text { una ordenación del atributo que se está } \\
\text { midiendo, pero sin informar nada respecto } \\
\text { a la magnitud de las diferencias entre los } \\
\text { números. Los números solo permiten } \\
\text { ordenar las categorías de la variable por } \\
\text { mayor o menor presencia del atributo } \\
\text { considerado }\end{array}$ & $\begin{array}{l}\text { La clase social de una } \\
\text { persona, que podría } \\
\text { escalarse como: } \\
1 \text { = baja } \\
2 \text { = media } \\
3 \text { = alta }\end{array}$ & $\begin{array}{l}\text { Mediana } \\
\text { Centiles } \\
\text { Correlación ordinal }\end{array}$ \\
\hline
\end{tabular}


J. Rodríguez-Rodríguez y M. Reguant-Álvarez. Calcular la fiabilidad de un cuestionario o escala mediante el SPSS: el coeficiente alfa de Cronbach

\begin{tabular}{|c|c|c|c|}
\hline Escala & Definición & Ejemplo & Estadísticos \\
\hline $\begin{array}{l}\text { De intervalo } \\
\text { (cuantitativa) }\end{array}$ & $\begin{array}{l}\text { En esta escala, la asignación alfanumérica } \\
\text { implica una cuantificación equidistante del } \\
\text { atributo, si bien la unidad de medida es } \\
\text { arbitraria dado que no define un valor cero } \\
\text { absoluto }\end{array}$ & $\begin{array}{l}\text { El coeficiente de } \\
\text { inteligencia. } \\
\text { El } 0 \text { no indica ausencia total } \\
\text { de inteligencia. La diferencia } \\
\text { entre un puntaje de } 100 \text { a } \\
120 \text { es la misma, en } \\
\text { términos de la variable, que } \\
\text { entre } 120 \text { y } 140 \text {. Los } \\
\text { intervalos son iguales }\end{array}$ & $\begin{array}{l}\text { Media aritmética } \\
\text { Desviación típica } \\
\text { Coeficiente } \\
\text { correlación } \\
\text { Pruebas de } \\
\text { contraste (t } \\
\text { Student...) }\end{array}$ \\
\hline $\begin{array}{l}\text { Razón } \\
\text { (cuantitativa) }\end{array}$ & $\begin{array}{l}\text { Permite clasificar (nominal), ordenar } \\
\text { (ordinal) y establecer la distancia (de } \\
\text { intervalo) de un atributo, con la presencia } \\
\text { de un cero absoluto que permite identificar } \\
\text { la falta de ese atributo }\end{array}$ & $\begin{array}{l}\text { Edad cronológica de una } \\
\text { persona }\end{array}$ & $\begin{array}{l}\text { Todos los cálculos } \\
\text { estadísticos }\end{array}$ \\
\hline
\end{tabular}

Fuente: Elaboración propia a partir de Behar (2008), López-Roldán y Fachelli (2015), Ormazábal et al. (2012).

Estas escalas de medida son las que van a permitir cuantificar los atributos de ese fenómeno, y para ello se creará un instrumento adecuado. De esta manera, cualquier instrumento de medida tiene que cumplir algunos requisitos para considerarse útil. Además de permitir cuantificar el consumo de agua, por ejemplo, un contador de agua es un recurso válido que también tiene que ser fiable. Fiabilidad y validez son dos términos que están relacionados, si bien en ocasiones puede confundirse su significado (Sabariego, 2004). La validez hace referencia a la calidad que posee un instrumento para ser útil en la medición de un atributo, es decir, si mide aquello que se supone que mide. Por ejemplo, un termómetro es un instrumento válido para medir la temperatura. La fiabilidad, o confiabilidad, en cambio, tiene que ver con la precisión de la medida, con la estabilidad en el tiempo. Es importante que la medición que se realiza a través de un instrumento -bien sea un cuestionario o un test-, sea válida y fiable.

\section{Justificación y objetivos del presente trabajo}

El cálculo del coeficiente de fiabilidad de un instrumento se puede llevar a cabo mediante diversos cálculos estadísticos, como se verá en el apartado 3.

Las investigaciones socioeducativas en las que se construyen, adaptan o utilizan instrumentos cuantitativos pueden beneficiarse del cálculo de fiabilidad. Los equipos de investigación deben constatar que los cuestionarios o tests que utilizan cumplen los estándares de fiabilidad y validez que la comunidad científica considera adecuados tanto para el ámbito de investigación como en la práctica aplicada. Así se manifiesta la Comisión Internacional de Tests (2014) en cuanto a la elaboración de nuevos instrumentos de medida. Por lo que respecta a instrumentos que ya se hayan validado, Barrios y Cosculluela (2013) y Campo-Arias y Oviedo (2008) señalan que es pertinente realizar los cálculos de fiabilidad dado que ésta puede variar según la población estudiada.

Así pues, este trabajo se plantea los siguientes objetivos: a) identificar los diferentes métodos para calcular la fiabilidad de un instrumento en el ámbito socioeducativo; b) exponer los pasos para calcular el estadístico alfa de Cronbach mediante el paquete estadístico SPSS, y c) ejemplificar el cálculo de la fiabilidad de una escala de actitudes tipo Likert. 


\section{El cálculo de la fiabilidad y su significación}

\subsection{Definición}

La fiabilidad tiene que ver con la precisión o consistencia de la medición (Barrios y Cosculluela, 2013; Brown, 2009). La fiabilidad "se concibe como la consistencia o estabilidad de las medidas cuando el proceso de medición se repite" (Prieto y Delgado, 2010, p. 67) o "aquella propiedad que valora la consistencia y precisión de la medida" (Barrios y Cosculluela, 2013, p. 75). Cuando una persona responde a un mismo cuestionario en diferentes momentos, bajo las mismas condiciones externas e internas, las variaciones en las puntuaciones recogidas serían indicativo del nivel de fiabilidad de la medida. No obstante, es imposible que se den las mismas condiciones externas e internas, así como demandar a las personas participantes que respondan las preguntas de un mismo cuestionario o test en múltiples ocasiones. Por este motivo, la medida del nivel de fiabilidad es hipotética. En definitiva, es importante recordar que la fiabilidad es una propiedad de las puntuaciones de un cuestionario o una escala para un grupo determinado de personas pertenecientes a una muestra concreta (Fan y Thompson, 2001).

En este sentido, la fiabilidad tiene que ver con la estimación de la característica verdadera de ese fenómeno (López-Roldán y Fachelli, 2015). Hace referencia a una estimación de ese atributo, a una aproximación de este, dado que la medición difícilmente recoge el verdadero valor, al menos en las Ciencias Humanas. Piénsese por ejemplo en la actitud del alumnado universitario de Educación Primaria en relación con la utilización del móvil en el aula. Si se elabora una escala de actitudes, que posea indicios de validez, esa estrategia de recogida de información proporcionará un valor aproximado de esa actitud - que vendrá dada por la respuesta del alumnado-, porque la actitud no es, en principio, directamente observable. Y hay que tener en cuenta que esta medición no está exenta de error, que puede ser de carácter sistemático o de carácter aleatorio (Barrios y Cosculluela, 2013; Drost, 2011). Por tanto, el valor observado es el valor verdadero más el posible error de medida - un error que puede deberse al método utilizado para medir, o bien debido a las características de las personas a las que se está valorando-.

Teniendo en cuenta estas afirmaciones, se hace evidente la importancia de contar con instrumentos válidos y fiables, a tenor de las implicaciones que pueden llegar a derivarse de su uso. Según LópezRoldán y Fachelli (2015), para aumentar la fiabilidad hay que disminuir los errores que se producen, y en un instrumento como un cuestionario o un test, esto se lleva a cabo a partir de:

- Aumentar el número de ítems o preguntas.

- Eliminar aquellos ítems que provocan diferentes respuestas a consecuencia de un redactado que se puede interpretar de maneras distintas.

- Controlar las condiciones en las que se administra la prueba, para que el contexto no interfiera en las respuestas de las personas.

- Proponer un redactado que facilite la comprensión de los ítems.

- Reducir las variables extrañas que puedan interferir en la respuesta a los ítems. 
- Presentar unas instrucciones iguales para todas las personas participantes.

- Tener un procedimiento de calificación y puntuación de las respuestas en consonancia con los ítems del cuestionario o test, $y$

- Realizar estudios longitudinales y desde diferentes equipos de investigación.

Existen diferentes métodos para estimar la fiabilidad de un instrumento, y cada uno de ellos tiene en cuenta aquellas cuestiones que afectan a la fiabilidad del mismo (Aiken, 2003; Barrios y Cosculluela, 2013; Hernández-Sampieri et al., 2014). Así, los métodos más comunes para calcular la fiabilidad son: coeficiente test-retest, las formas paralelas, o la consistencia interna. No obstante, en ocasiones puede requerirse utilizar otros métodos, como por ejemplo el coeficiente Omega (Viladrich et al., 2017).

El coeficiente test-retest -o coeficiente de estabilidad- establece la correlación entre las respuestas que se producen de aplicar el mismo instrumento en dos momentos distintos. Al utilizarse el mismo instrumento de medida, se pueden producir errores debidos a la influencia de las condiciones ambientales y personales. Ahora bien, este cálculo se ve afectado por el lapso de tiempo entre el test y el retest, de manera que, a mayor intervalo de tiempo, mayor probabilidad de error (Aiken, 2003; Prieto y Delgado, 2010).

El coeficiente de formas paralelas - o coeficiente de equivalencia - tiene en cuenta el aprendizaje que realizan las personas a las que se administra el cuestionario o test en un primer momento y después de un tiempo de la primera administración (Aiken, 2003; Prieto y Delgado, 2010). Por tanto, este coeficiente se calcula a partir de aplicar una forma paralela a la prueba inicial, es decir, se administra una forma $\mathrm{A}$ en el momento inicial, $\mathrm{y}$ una forma $\mathrm{B}$ equivalente, en el momento final.

El coeficiente de consistencia interna, que es el que se va a detallar en este artículo, se utiliza cuando no es posible una segunda aplicación a un mismo grupo de sujetos o cuando la elaboración de una forma paralela del instrumento resulta costosa o difícil para el equipo de investigación. En este caso se puede utilizar la división por mitades, el método Kuder-Richarson o bien el coeficiente alfa de Cronbach (Aiken, 2003; Barrios y Cosculluela, 2013; Celina y Campo, 2005; Cronbach, 1951; Prieto y Delgado, 2010). Esto es, dividir el instrumento de medida en dos partes equivalentes y comparar ambos resultados, o bien hacer la comparación de todos los ítems entre sí.

\subsection{Cálculo}

El coeficiente alfa de Cronbach es una fórmula general para estimar la fiabilidad de un instrumento en el que la respuesta a los ítems es dicotómica o tiene más de dos valores (Aiken, 2003; Cortina, 1993), como por ejemplo en una escala de actitudes con respuesta de tipo Likert. Dado que es uno de los tipos de coeficiente de consistencia interna, el alfa de Cronbach expresa esta consistencia interna a partir de la covariación entre los ítems del cuestionario o test, de manera que cuanto mayor es la covariación, mayor puntuación alfa (Barrios y Cosculluela, 2013). Sin embargo, a pesar de la elevada popularidad del coeficiente alfa, algunos autores (Brown, 2009; Cortina, 1993; Drost, 2011; Schmitt, 1996; Sijtsma, 2009; Viladrich et al., 2017) señalan ciertas cuestiones en relación con este coeficiente, que puede subestimar o sobreestimar la fiabilidad del instrumento, y que hay que tener 
presentes para una adecuada interpretación y utilización del mismo -influencia del número de ítems del cuestionario o la multidimensionalidad del mismo, entre otras-. No obstante, Elosua y Zumbo (2008) apuntan diversos estudios que muestran cómo, en el caso de las escalas tipo Likert, los resultados son más legítimos cuando se dan cinco o más categorías de respuesta y cuando existe un número suficiente de sujetos.

Si bien existen diferentes fórmulas para calcular el coeficiente alfa de Cronbach, la más utilizada es la siguiente (Aiken, 2003; Barrios y Cosculluela, 2013):

$$
\alpha=\frac{k\left(1-\sum s_{i}^{2} / s_{t}^{2}\right)}{k-1}
$$

Así, $k$ es el número de ítems del instrumento, $s_{i}^{2}$ la varianza de las puntuaciones en el ítem i, y $s_{t}^{2}$ la varianza de las puntuaciones totales del cuestionario o test.

Afortunadamente, en la actualidad existen programas estadísticos que facilitan la tarea de calcular la fiabilidad de un instrumento. El Statistical Package for the Social Sciences (SPSS versión 25) permite calcular el coeficiente alfa de Cronbach de manera sencilla, siguiendo los pasos que se detallan a continuación:

1. En primer lugar, seleccionar la opción Analizar del menú principal. Este apartado permite calcular estadísticos descriptivos, correlaciones, reducción de dimensiones, etc. Dentro de la opción Analizar, seleccionar Escala, y a continuación Análisis de la fiabilidad.

2. Seleccionar los ítems del cuestionario o test que queramos analizar y pasarlos a la casilla Elementos pulsando la flecha en horizontal.

3. A continuación, en la parte superior derecha, pulsar Estadísticos y seleccionar aquellos que interesan para el estudio:

- Descriptivos: elemento, escala y escala si se elimina el elemento.

- Inter-elementos: para correlaciones y covarianzas.

- Resúmenes: medias, varianzas, covarianzas y correlaciones

4. Y pulsamos Continuar.

5. Eso retorna a la pantalla anterior - donde se habían seleccionados los ítems del cuestionarioy puede apreciarse que en la parte inferior, la casilla Modelo tiene seleccionado por defecto Alfa. Pulsar la tecla Aceptar y aparecen los resultados en el Visor de resultados.

Conviene tener presente que el SPSS no proporciona de forma directa los intervalos de confianza en el cálculo del estadístico, y esto sería recomendable ya que la fiabilidad se ve afectada por el error muestral, por un lado, y por el hecho que los instrumentos de medida no tienen una fiabilidad absoluta (Ledesma, 2004; Merino-Soto, 2016). Por tanto, la ausencia de estos intervalos de confianza limita la interpretación del estadístico. 


\section{Figura 1}

Cuadro de diálogo para realizar el análisis de fiabilidad

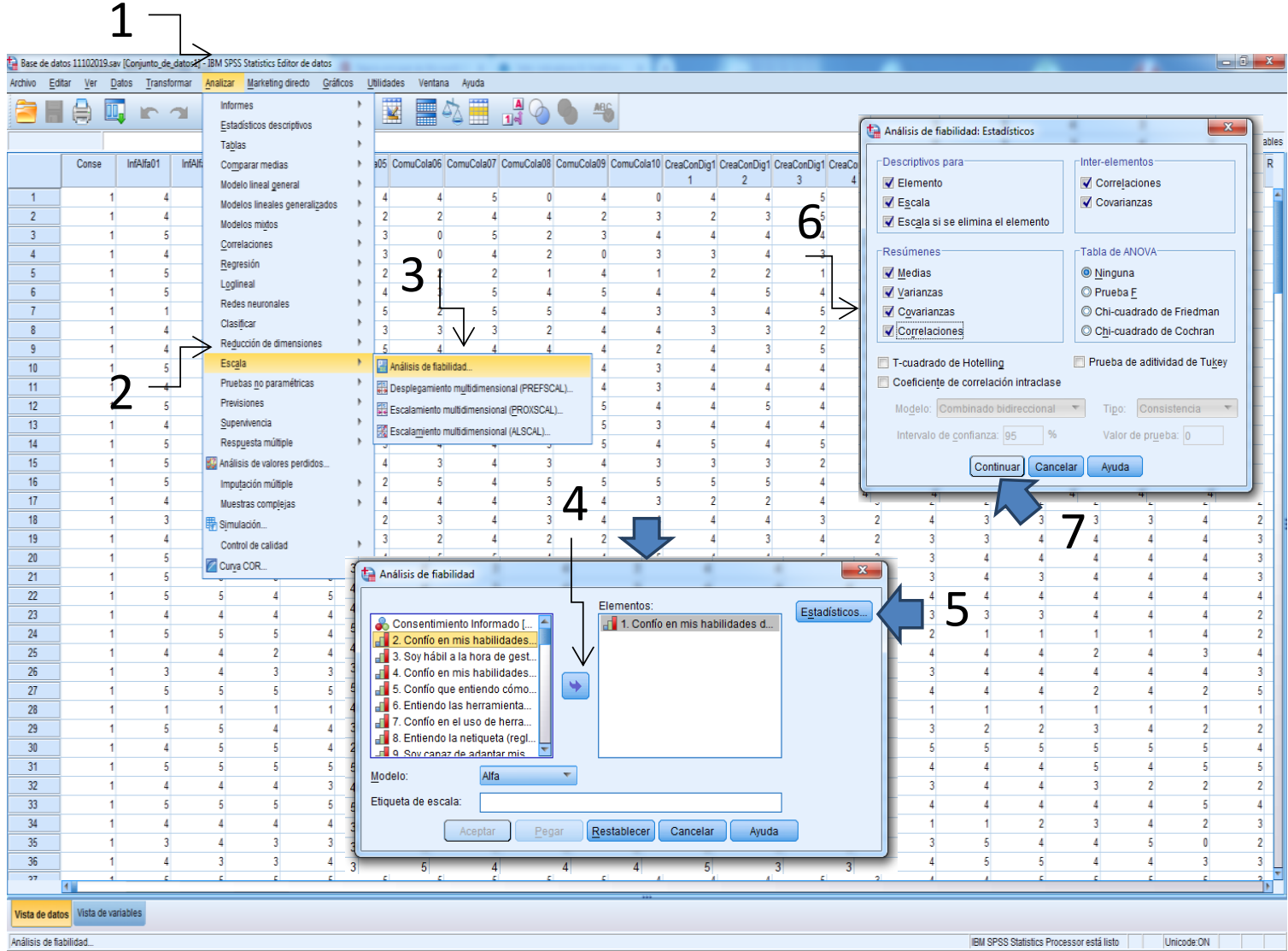

Se van a utilizar, a modo de ejemplo, una serie datos procedentes de un hipotético estudio sobre actitudes del alumnado universitario hacia el deporte. El objetivo del instrumento podría ser: medir las actitudes y significado que otorgan los estudiantes universitarios a la práctica deportiva. Pensamos en un instrumento de 22 ítems tipo Likert, con un rango de respuesta de 1 a 5, donde 1 significa "En total desacuerdo" y 5 "Totalmente de acuerdo". Para ilustrar, el contenido de algún ítem de este cuestionario simulado:

1. En caso de tener una pareja aficionada al deporte, la apoyaría para que lo practicara.

2. Me agrada relacionarme con personas que practican cualquier tipo de deporte.

3. Me desagrada mucho el carácter competitivo que suelen tener las/los deportistas.

En la Tabla 2 puede verse el índice de fiabilidad global de este supuesto cuestionario y el número de ítems del mismo. 
Tabla 2

Estadísticas de fiabilidad para el cuestionario

\begin{tabular}{ccc}
\hline & $\begin{array}{c}\text { Alfa de Cronbach basada } \\
\text { en elementos } \\
\text { estandarizados }\end{array}$ & N de elementos \\
\hline Alfa de Cronbach &, 742 & 22 \\
\hline, 758 & &
\end{tabular}

Los datos de esta simulación señalan que ese cuestionario de 22 elementos tiene en conjunto una fiabilidad de 0,758 , resultando un valor aceptable teniendo en cuenta el margen que señalan las autoras y autores habitualmente referenciadas - véase siguiente apartado relativo a la Significación del coeficiente-.

Otros datos que proporciona el SPSS son la media y desviación típica de cada uno de los ítems, tal y como se señala en la Tabla 3. La desviación típica o estándar refleja el grado de distancia entre las puntuaciones individuales y la media (Hurtado y Hurtado, 2015). Así, se observa que la pregunta 4 es la que tiene mayor desviación típica.

Tabla 3

Estadísticos para los ítems del cuestionario

\begin{tabular}{lccc}
\hline & Media & $\begin{array}{c}\text { Desviación } \\
\text { estándar }\end{array}$ & $\mathrm{N}$ \\
\hline ítem 1 & 1,74 & 1,098 & 110 \\
ítem 2 & 1,91 & 1,113 & 110 \\
ítem 3 & 4,61 &, 767 & 110 \\
ítem 4 & 3,48 & 1,155 & 110 \\
ítem 5 & 4,63 &, 619 & 110 \\
ítem 6 & 4,28 &, 978 & 110 \\
ítem 7 & 4,20 &, 965 & 110 \\
$\ldots$ & & & \\
\hline
\end{tabular}

Las correlaciones entre los diferentes ítems del cuestionario - ver Tabla 4- señalan la relación de cada una de las preguntas consigo misma -en diagonal- y con las demás. Por ejemplo, el ítem 1 tiene una correlación positiva de 0,072 con el ítem 4, y negativa de 0,211 con el ítem 3.

\section{Tabla 4}

Correlación entre elementos (ítems) del cuestionario

\begin{tabular}{cccccccc}
\hline & ítem 1 & ítem 2 & ítem 3 & ítem 4 & ítem 5 & ítem 6 & ítem 7 \\
\hline ítem 1 & 1,000 &, 416 &,- 211 &, 072 &, 016 &,- 127 &,- 244 \\
ítem 2 &, 416 & 1,000 &, 033 &,- 037 &, 070 &,- 044 &,- 026 \\
ítem 3 &,- 211 &, 033 & 1,000 &,- 044 &, 347 &, 515 &, 503 \\
ítem 4 &, 072 &,- 037 &,- 044 & 1,000 &, 164 &,- 113 &,- 030 \\
ítem 5 &, 016 &, 070 &, 347 &, 164 & 1,000 &, 312 &, 280 \\
ítem 6 &,- 127 &,- 044 &, 515 &,- 113 &, 312 & 1,000 &, 766 \\
ítem 7 &,- 244 &,- 026 &, 503 &,- 030 &, 280 &, 766 & 1,000 \\
ítem 8 &,- 021 &,- 039 &, 013 &, 165 &, 161 &,- 147 &,- 112 \\
ítem 9 &, 302 &, 050 &,- 361 &, 079 &,- 098 &,- 173 &,- 209 \\
\hline
\end{tabular}


Finalmente, en la Tabla 5 puede verse un resumen de los estadísticos del cuestionario en su conjunto, y recoge el coeficiente alfa de Cronbach cuando se suprime cada uno de los ítems. Se observa que eliminando el ítem 6, la fiabilidad global del instrumento - sin el ítem- pasa de 0,758 a 0,769, y si se elimina el ítem 7, la fiabilidad global es de 0,769 o el ítem 3 en el que la fiabilidad aumenta a 0,763, lo que quiere decir que estos tres ítems no aportan demasiado a la consistencia interna del conjunto de la prueba.

\section{Tabla 5}

Estadísticas de total de elementos para el cuestionario

\begin{tabular}{ccccc}
\hline & $\begin{array}{c}\text { Media de escala si el } \\
\text { elemento se ha } \\
\text { suprimido }\end{array}$ & $\begin{array}{c}\text { Varianza de escala si } \\
\text { el elemento se ha } \\
\text { suprimido }\end{array}$ & $\begin{array}{c}\text { Correlación total de } \\
\text { elementos corregida }\end{array}$ & $\begin{array}{c}\text { Alfa de Cronbach si } \\
\text { el elemento se ha } \\
\text { suprimido }\end{array}$ \\
\hline ítem 1 & 101,35 & 129,311 &, 373 &, 746 \\
ítem 2 & 101,17 & 133,612 &, 193 &, 756 \\
ítem 3 & 98,47 & 139,628 &,- 022 &, 763 \\
ítem 4 & 99,60 & 129,912 &, 326 &, 753 \\
ítem 5 & 98,45 & 135,553 &, 270 &, 769 \\
ítem 6 & 98,80 & 141,336 &,- 106 &, 768 \\
ítem 7 & 98,88 & 140,968 &,- 091 &, 752 \\
ítem 8 & 99,13 & 133,396 &, 258 &, 755 \\
ítem 9 & 100,66 & 132,812 &, 203 & , \\
\hline
\end{tabular}

En estos tres casos, hay que hacer análisis complementarios tanto de orden estadístico, como teóricos que nos permitan decidir si vale la pena mantener el ítem, reformularlo o eliminarlo.

\subsection{Significación}

Una vez se calcula la fiabilidad de un instrumento y se obtiene un valor numérico asignado a ese índice de fiabilidad, el siguiente paso es dar significado a ese valor. La fiabilidad se expresa mediante un número decimal positivo que oscila entre 0,00 y 1,00 , desde una falta de fiabilidad hasta una fiabilidad perfecta (Castañeda et al., 2010).

Ahora bien, no solo resulta importante calcular el valor numérico, sino que además hay que ver qué significa ese valor en cuestión. En este sentido, cabe destacar que no existe un criterio aceptado como adecuado por toda la comunidad científica (Barrios y Cosculluela, 2013; Hernández-Sampieri et al., 2014). Por otra parte, se necesita contextualizar los resultados de la medición, es decir, en qué escenario se está utilizando el instrumento en cuestión para interpretar y valorar adecuadamente el cálculo de la fiabilidad (Aiken, 2003; Drost, 2011). El texto clásico de Nunnally (1978) señala que la puntuación mínima aceptable se situaría en 0,70 . Sin embargo, si lo que se pretende es comparar las puntuaciones de dos personas, o comparar las puntuaciones de una persona en dos instrumentos, como pasa en contextos escolares o clínicos, entonces se requieren valores iguales o superiores a 0,80 o 0,90. Prieto y Delgado (2010) consideran que si las decisiones que se derivan de las puntuaciones de un instrumento tienen una repercusión muy significativa en la persona, entonces el coeficiente alfa debería ser igual o superior a 0,90. En cualquier caso, estos mismos autores señalan que estas orientaciones se deben tomar con cautela debido a los diferentes tipos de errores subyacentes a las diversas maneras de calcular el índice de fiabilidad. 
Teniendo en cuenta estos matices, Barrios y Cosculluela (2013) concluyen que la fiabilidad adecuada oscila entre 0,70 y 0,95 ; y señalan que los valores muy cercanos a 1 pueden implicar ítems redundantes que no proporcionan información relevante sobre los atributos que se intenta medir.

\section{Conclusiones}

En el área socioeducativa, está ampliamente difundido el uso de cuestionarios como instrumento de consulta, por lo que es de suma importancia garantizar que estos poseen las condiciones técnicas necesarias.

La fiabilidad constituye una de las principales cualidades técnicas de los instrumentos de medida, incluso muy ligada a la validez de contenido (Pérez Juste et al., 2009). La fiabilidad nos habla de precisión y constancia en la información recogida, lo que resulta indispensable para hacer investigaciones con un nivel de rigor aceptable.

Son varios los procedimientos posibles para calcular la fiabilidad de una prueba, como ha podido observarse en el presente trabajo, el cálculo del coeficiente de fiabilidad alfa de Cronbach, mediante el paquete estadístico SPSS, es un procedimiento relativamente sencillo y rápido. De esta manera, este artículo representa un documento de soporte metodológico para el alumnado y también para las personas que realizan investigaciones más amplias en el contexto socioeducativo.

\section{Referencias}

Aiken, L. R. (2003). Tests psicológicos y evaluación. Pearson Educación.

Barrios, M., y Cosculluela, A. (2013). Fiabilidad. En J. Meneses (coord.), Psicometría (pp. 75-140). UOC.

Behar, D. S. (2008). Introducción a la Metodología de la Investigación. Editorial Shalom.

Brown, T. A. (2009). Confirmatory Factor Analysis for Applied Research. The Guilford Press.

Campo-Arias, A., y Oviedo, H. C. (2008). Propiedades psicométricas de una escala: la consistencia interna. Revista de Salud Pública, 10(5), 831-839. https://doi.org/10.1590/s0124-00642008000500015

Castañeda, M. B., Cabrera, A. F., Navarro, Y., y De Vires, W. (2010). Procesamiento de datos y análisis estadístico utilizando SPSS. EDIPUCRS.

Celina, H., y Campo, A. (2005). Aproximación al uso del coeficiente alfa de Cronbach. Revista colombiana de psiquiatría, XXXIV(4), 572-580. https://www.redalyc.org/pdf/806/80634409.pdf

Comisión Internacional de Tests (2014). El uso de los tests y otros instrumentos de evaluación en investigación. Consejo General de la Psicología. https://www.cop.es/pdf/ITC2015-Investigacion.pdf

Cortina, J. M. (1993). What is coefficient alpha? An examination of theory and applications. Journal of Applied Psychology, 78(1), 98-104. https://doi.org/10.1037/0021-9010.78.1.98 
J. Rodríguez-Rodríguez y M. Reguant-Álvarez. Calcular la fiabilidad de un cuestionario o escala mediante el SPSS: el coeficiente alfa de Cronbach

Cronbach, L. J. (1951). Coefficient alpha and the internal structure of tests. Psychometrika, 16(3), 297-334. https://doi.org/10.1007/BF02310555

Drost, E. A. (2011). Validity and Reliability in Social Science Research. Education Research and Perspectives, $38(1), 105-123$.

Elosua, P., y Zumbo, B. D. (2008). Coeficientes de fiabilidad para escalas de respuesta categórica ordenada. Psicothema, 20(4), 896-901. http://www.psicothema.com/psicothema.asp?id=3572

Fan, X., y Thompson, B. (2001). Confidence intervals about score reliability coefficients, please: An EPM guidelines editorial. Educational and Psychological Measurement, 61(4), 517-531. https://doi.org/10.1177/0013164401614001

Hernández-Sampieri, R., Fernández, C., y Baptista, M. P. (2014). Metodología de la investigación. McGrawHill.

Hurtado, A. L., y Hurtado, C. L. (2015). La toma de decisiones e investigación educativa con SPSS. Qartuppi. https://doi.org/10.29410/qtp.15.03

Ledesma, R. (2004). AlphaCl: un programa de cálculo de intervalos de confianza para el coeficiente alfa de Cronbach. Psico-USF, 9(1), 31-37. https://doi.org/10.1590/s1413-82712004000100005

López-Roldán, P., y Fachelli, S. (2015). Metodología de la investigación social cuantitativa. UAB.

Merino-Soto, C. (2016). Diferencias entre coeficientes alfa de Cronbach, con muestras y partes pequeñas: Un programa VB. Anales de psicología, 32(2), 587-588. https://doi.org/10.6018/analesps.32.2.203841

Nunnally, J. C. (1978). Psychometric theory. McGraw-Hill.

Ormazábal, F. J., Vilà, R., Mateo, M., Torrado, M., Berlanga, V., Del Barrio, J., y Ruiz, A. (2012). Introducció a l'estadística aplicada a l'educació. Conceptes bàsics. Universitat de Barcelona. http://hdl.handle.net/2445/21376

Pérez Juste, R., García Llamas, J., Gil, J., y Galán, A. (2009). Estadística aplicada a la Educación. Pearson.

Prieto, G., y Delgado, A. R. (2010). Fiabilidad y validez. Papeles del Psicólogo, 31(1), 67-74. http://www.papelesdelpsicologo.es/pdf/1797.pdf

Reguant, M., y Martínez-Olmo, F. (2014). Operacionalización de conceptos/variables. Dipòsit Digital de la UB. http://hdl.handle.net/2445/57883

Sabariego, M. (2004). El proceso de investigación (parte 2). En R. Bisquerra (coord.), Metodología de la investigación educativa (pp. 127-163). La Muralla.

Schmitt, N. (1996). Uses and Abuses of Coefficient Alpha. Psychological Assessment, 8(4), 350-353. https://doi.org/10.1037/1040-3590.8.4.350 
J. Rodríguez-Rodríguez y M. Reguant-Álvarez. Calcular la fiabilidad de un cuestionario o escala mediante el SPSS: el coeficiente alfa de Cronbach

Sijtsma, K. (2009). On the use, the misuse, and the very limited usefulness of Cronbach's Alpha. Psychometrika, 74(1), 107-120. http://doi.org/10.1007/s11336-008-9101-0

Viladrich, C., Angulo-Brunet, A., y Doval, E. (2017). Un viaje alrededor de alfa y omega para estimar la fiabilidad de consistencia interna. Anales de psicología, 33(3), 755-782. https://doi.org/10.6018/analesps.33.3.268401 